\title{
Extracellular signal-regulated kinase inhibition is required for methanol extract of Smilax china L.-induced apoptosis through death receptor 5 in human oral mucoepidermoid carcinoma cells
}

\author{
HYUN-JU YU ${ }^{1}$, JI-AE SHIN ${ }^{1}$, SYNG-OOK LEE $^{2}$, KI HAN KWON ${ }^{3}$ and SUNG-DAE CHO ${ }^{1}$ \\ ${ }^{1}$ Department of Oral Pathology, School of Dentistry and Institute of Oral Bioscience, Chonbuk National University, \\ Jeonju, North Jeolla 561-756, Republic of Korea; ${ }^{2}$ Department of Veterinary Physiology and Pharmacology, \\ Texas A\&M University, College Station, TX 77843, USA; ${ }^{3}$ Department of Food Science and Nutrition, \\ College of Health, Welfare and Education, Gwangju University, Gwangju 503-703, Republic of Korea
}

Received June 4, 2013; Accepted November 18, 2013

DOI: $10.3892 / \mathrm{mmr} .2013 .1826$

\begin{abstract}
Smilax china L., a well-known Chinese traditional medicine, has been used as an anti-inflammatory, anti-cancer and analgesic agent, but its role has not yet been fully elucidated in oral mucoepidermoid carcinoma (MEC). The present study focused on addressing the anticancer activity and molecular mechanism of methanol extract of Smilax china L. (MESC) in MC-3 human oral MEC cells. The results indicated that MESC inhibited cell growth and induced apoptosis in MC-3 cells. These observations were found to correlate with increases in truncated $\mathrm{BH} 3$ interacting-domain death agonist and B-cell lymphoma 2 (Bcl-2) interacting mediator of cell death, but not Bcl-2 homologous antagonist killer, Bcl-2-associated $\mathrm{X}$ protein, $\mathrm{Bcl}-2$, $\mathrm{B}$-cell lymphoma-extra large and induced myeloid leukemia cell differentiation protein levels. MESC also damaged the mitochondrial membrane potential, cleaved caspase- 8 protein and increased death receptor 5 (DR5) protein levels by enhancing the stability of DR5 protein. Furthermore, MESC affected the phosphorylation of extracellular signal-regulated kinase (ERK) only, and did not affect c-Jun N-terminal kinase or p38 phosphorylation. Co-treatment with MESC and an ERK inhibitor (PD98059) significantly increased the expression of DR5 to induce apoptosis in MC-3 cells. Therefore, these results suggest that MESC may induce
\end{abstract}

Correspondence to: Professor Sung-Dae Cho, Room 319, Duckjindong 1ga, Department of Oral Pathology, School of Dentistry and Institute of Oral Bioscience, Chonbuk National University, Jeonju, North Jeolla 561-756, Republic of Korea

E-mail: efiwdsc@chonbuk.ac.kr

Key words: Smilax china L., mucoepidermoid carcinoma, extracellular signal-related kinase, death receptor 5, BH3 interacting-domain death agonist, B-cell lymphoma 2 interacting mediator of cell death apoptosis via the ERK pathway and may be a potential anticancer drug candidate against human oral MEC.

\section{Introduction}

Oral cancer ranks among the ten most frequently diagnosed cancers worldwide and has one of the lowest five-year survival rates $(1,2)$. Mucoepidermoid carcinoma (MEC), a type of oral cancer, is the most common malignant salivary gland tumor, usually affecting the parotid and minor salivary glands of adults. Due to the phenotypic and biological heterogeneity of MECs, several studies have attempted to identify potential biomarkers and therapeutic strategies to improve clinical assessment (3-6).

Naturally-occurring products, derived from plant sources, are used as traditional medicines due to their potential chemotherapeutic activity $(7,8)$. Smilax china L. is a small vine that grows in the southern parts of China. It has a long history of indigenous use and has been widely used in Chinese traditional medicine for treatment of diverse diseases, particularly for acute and chronic pelvic inflammation $(9,10)$. Recently, several studies have reported that Smilax china L.-derived compounds have anticancer activities in various cancer cell lines, including cervical, lung, gastric and breast cancers (11-13). However, the apoptotic effect of Smilax china L. in MEC has not been thoroughly investigated.

Mitogen-activated protein kinase (MAPK) cascades have been reported to be central in integrating the signals from a diverse group of extracellular stimuli to the nucleus, where activation of specific transcription factors elicits cellular responses by the regulation of gene expression (14). Three major MAPK pathways have been identified in mammals, extracellular signal-regulated kinases (ERKs), stress-activated protein kinases/c-Jun N-terminal kinases (JNKs) and p38 MAPKs. Among them, the ERK1/2 pathway contributes to survival signaling in various tumors and inhibition of the ERK1/2 signaling pathway may be more effective for inducing tumor cell death than JNK and p38 (15). In the present study, the antiproliferative effects of the methanol extract of 
Smilax china L. (MESC), and the associated signaling pathways in MC-3 human oral MEC cells, were investigated.

\section{Materials and methods}

Reagents and antibodies. MESC was provided by Professor Ki-Han Kwon (Kwangju University, Kwangju, Korea). Dulbecco's modified Eagle's medium (DMEM), fetal bovine serum (FBS), 100X antibiotic solution, trypsin and Dulbecco's phosphate-buffered saline (PBS) were obtained from WelGENE Inc. (Daegu, Korea). The actin antibody was purchased from Santa Cruz Biotechnology, Inc. (Santa Cruz, CA, USA). Death receptor 4 (DR4) antibody was obtained from R\&D Systems Inc. (Minneapolis, MN, USA). Antibodies against B-cell lymphoma 2 (Bcl-2) interacting mediator of cell death (Bim), truncated $\mathrm{BH} 3$ interacting-domain death agonist (t-Bid), Bcl-2 homologous antagonist killer, Bcl-2-associated $\mathrm{X}$ protein, Bcl-2, B-cell lymphoma-extra large, myeloid leukemia cell differentiation protein, cleaved caspase-8, p-ERK, ERK, p-JNK, JNK, p-p38 and p38 were supplied by Cell Signaling Technology, Inc. (Danvers, MA, USA). DAPI and cycloheximide (CHX) were acquired from Sigma-Aldrich (St. Louis, MO, USA). PD98059 was provided by Calbiochem Technology (San Diego, CA, USA).

Cell culture and drug treatment. MC-3 human MEC cells were provided by Professor Wu Junzheng (Forth Military Medical University, Xi'an, China). MC-3 cells were cultured in DMEM supplemented with $10 \%$ FBS and $100 \mathrm{U} / \mathrm{ml}$ each of penicillin and streptomycin in a humidified atmosphere containing $5 \% \mathrm{CO}_{2}$ at $37^{\circ} \mathrm{C}$. An equal number of cells were seeded and allowed to attach. When the cells reached 50\% confluency, they were treated with dimethylsulfoxide (DMSO) or MESC (25, 50 and $100 \mu \mathrm{g} / \mathrm{ml})$. MESC was dissolved in $0.1 \%$ DMSO (vehicle control).

Cell viability assay. The MC- 3 cells were treated with 25,50 and $100 \mu \mathrm{g} / \mathrm{ml}$ MESC for $24 \mathrm{~h}$ and the number of viable cells was counted using a hematocytometer (Neubauer Chamber, Thermo Fisher Scientific, Waltham, MA, USA) with trypan blue $(0.4 \%)$. Each experiment was conducted in triplicate and the results were expressed as the mean \pm standard deviation for each treatment group.

DAPI staining. Apoptotic cell death was determined morphologically using the fluorescent nuclear dye, DAPI. MC-3 cells were harvested by trypsinization and fixed in $100 \%$ ethanol overnight at $-20^{\circ} \mathrm{C}$. The cells were re-suspended in PBS, deposited on poly-L-lysin-coated slides and stained with DAPI. The cell morphology was observed under a fluorescence microscope (Axio Imager.M2; Carl Zeiss Co. Ltd., Seoul, Republic of Korea).

Western blot analysis. Whole-cell lysates were extracted with lysis buffer and quantified using the DC Protein Assay kit (Bio-Rad, Hercules, CA, USA). Equal quantities of protein from each sample were mixed with $5 \mathrm{X}$ loading buffer and heated at $95^{\circ} \mathrm{C}$ for $5 \mathrm{~min}$. Equal quantities of protein were separated by SDS-PAGE and transferred onto a polyvinylidene fluoride membrane (Bio-Rad). The membranes were blocked with 5\% skimmed milk in Tris-buffered saline with Tween 20 (TBST) buffer at room temperature for $2 \mathrm{~h}$, washed with TBST and maintained overnight at $4^{\circ} \mathrm{C}$ with primary antibody. The membranes were then washed with TBST, followed by incubation with horseradish peroxidase-conjugated secondary antibody (Santa Cruz Biotechnology, Inc.) at room temperature for $2 \mathrm{~h}$. Following an additional wash with TBST, antibody-bound proteins were detected using an Enhanced Chemiluminescence Western Blotting Luminol Reagent (Santa Cruz Biotechnology, Inc.).

JC-1 assay. Alterations in mitochondrial membrane potential (MMP) were measured using the Mitochondrial Membrane Potential Detection kit (Stratagene, La Jolla, CA, USA). MC-3 cells were treated with MESC and incubated with $1 \mathrm{X}$ JC-1 reagent for $15 \mathrm{~min}$ at $37^{\circ} \mathrm{C}$ in a $5 \% \mathrm{CO}_{2}$ incubator. Cells were then washed with PBS (3X). JC-1 fluorescence was measured using a microplate reader (Plate CHAMELEON; Hidex, Turku, Finland).

Reverse transcription-polymerase chain reaction (RT-PCR). Total RNA was extracted from the cells using an easy-BLUE Total RNA Extraction kit (Intron, Seoul, Korea). Subsequently, $1 \mu \mathrm{g}$ RNA was used to synthesize the cDNA using the Reverse Transcription system (Promega, Madison, WI, USA). The PCR conditions of DR5 were as follows: 30 cycles; $1 \mathrm{~min}$ at $95^{\circ} \mathrm{C}$, $1 \mathrm{~min}$ at $57.8^{\circ} \mathrm{C}$ and $1 \mathrm{~min} 30 \mathrm{sec}$ at $72^{\circ} \mathrm{C}$. The PCR conditions of $\beta$-actin were as follows: 28 cycles; $1 \mathrm{~min}$ at $95^{\circ} \mathrm{C}, 1 \mathrm{~min}$ at $60^{\circ} \mathrm{C}$ and $1 \mathrm{~min} 30 \mathrm{sec}$ at $72^{\circ} \mathrm{C}$. The primer sequences used were as follows: DR5 Sense: 5'-ATG AGA TCG TGA GTA TCT TGC AGC-3' and antisense: 5'-TGA CCC ACT TTA TCA GDA TCG TGT-3' for DR5; and sense: 5'-GTG GGG CGC CCC AGG CAC CA-3' and antisense: 5'-CTC CTT AAT GTC ACG CAC GAT TTC-3' for $\beta$-actin. The PCR products were analyzed by $2 \%$ agarose gel electrophoresis.

Statistical analysis. The data were assessed for statistical significance using Student's t-test. A value of $\mathrm{P}<0.05$ compared with the vehicle control was considered to indicate a statistically significant difference.

\section{Results}

Effect of MESC on cell viability and apoptosis in MC-3 cells. To investigate the growth-inhibitory effect of MESC in MC-3 cells, an optic microscope was used and a trypan blue exclusion assay was performed to observe morphological changes. The results indicated that cells were detached and cell viability was decreased by MESC in a dose-dependent manner (Fig. 1A and B). To examine whether the antiproliferative effect of MESC was associated with apoptotic events, DAPI staining was performed to evaluate nuclear fragmentation and condensation (which is characteristic of cells undergoing apoptosis). As shown in Fig. 1C, MESC increased the number of condensed and fragmented nuclei compared with DMSO (vehicle control). These observations suggest that MESC can induce apoptosis to reduce the viability of MC-3 cells.

MESC activates caspase- 8 and increases $t$-Bid and Bim expression to damage MMP. It has been reported that a 
A

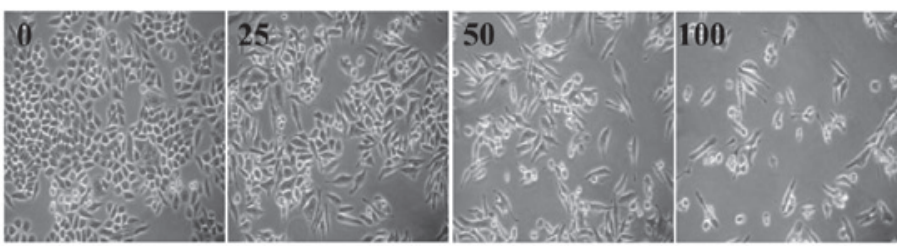

C

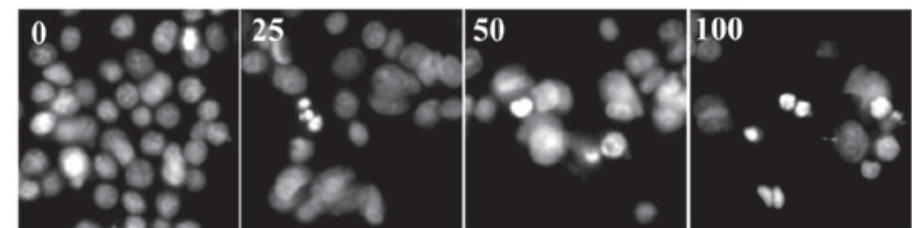

B
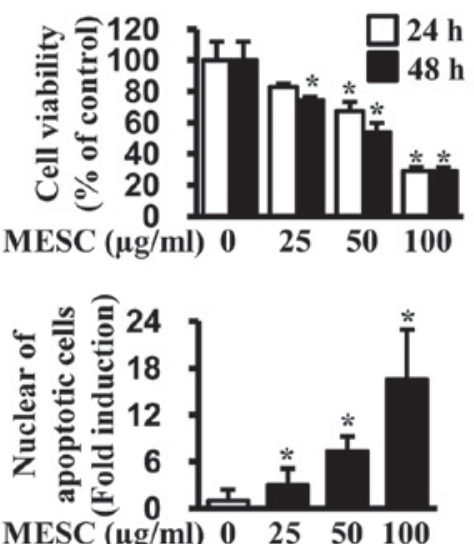

Figure 1. The effect of MESC on cell viability and apoptosis in MC-3 cells. MC-3 cells were treated with dimethylsulfoxide (vehicle control) or various doses of $\operatorname{MESC}(25,50$ and $100 \mu \mathrm{g} / \mathrm{ml}$ ) for $24 \mathrm{~h}$. (A) Cell morphology was observed by optical microscopy (magnification, x200). (B) Cell viability was determined using a trypan blue exclusion assay in MC-3 cells; bars represent the mean \pm SD of triplicate experiments. (C) Fluorescence microscopy images of the DAPI-stained MC-3 cells. The apoptotic cells were counted and data are presented as the mean \pm SD of triplicate experiments. "P $<0.05$, vs. the vehicle control group. MESC, methanol extract of Smilax china L.; SD, standard deviation.

A

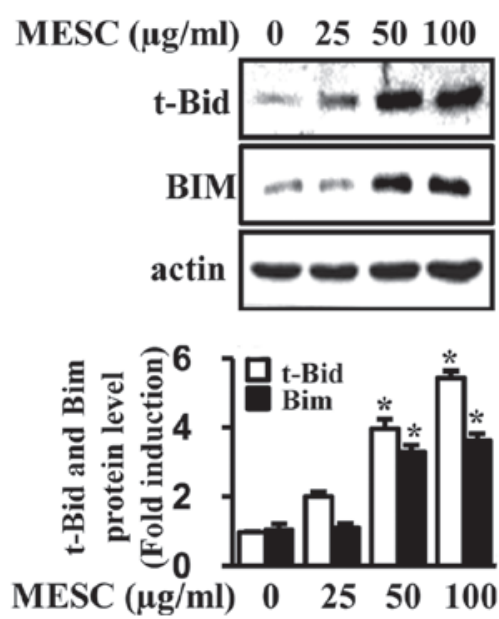

C

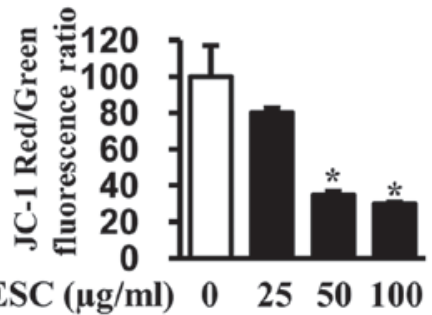

B

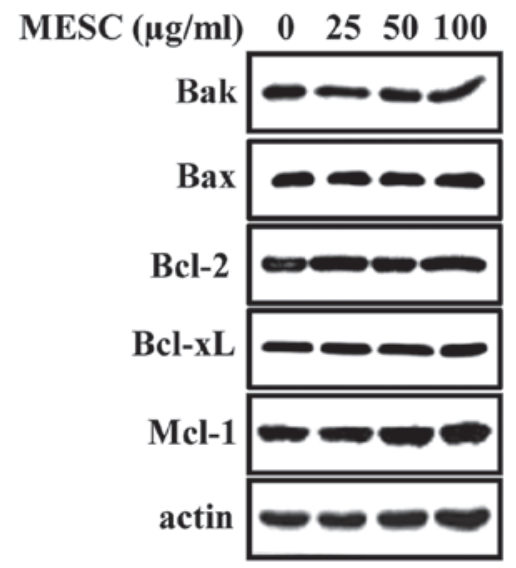

D

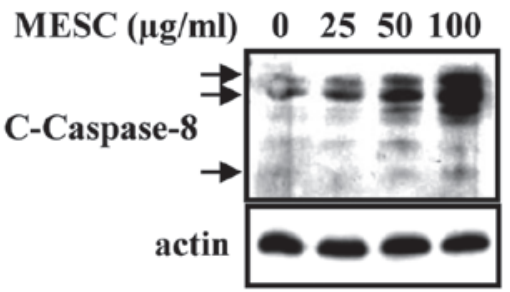

Figure 2. Induction of t-Bid, Bim and cleaved caspase- 8 to damage MMP by MESC. MC-3 cells were treated with dimethylsulfoxide (vehicle control) or various doses of $\operatorname{MESC}(25,50$ and $100 \mu \mathrm{g} / \mathrm{ml}$ ) for $24 \mathrm{~h}$. (A and B) Expression of various members of the Bcl-2 protein family (t-Bid, Bim, Bak, Bax, Bcl-2, $\mathrm{Bcl}-\mathrm{xl}$ and Mcl-1) was detected by western blot analysis. Protein levels were normalized to actin. The expression of t-Bid and Bim proteins were confirmed in triplicate with columnar graphs expressing the mean $\pm \mathrm{SD}$. (C) MMP was measured using the JC-1 assay kit and data are presented as the mean \pm SD of triplicate experiments. (D) Cleaved caspase- 8 was detected using western blot analysis in MC- 3 cells. "P<0.05, vs. the vehicle control group. MMP, mitochrondrial membrane potential; MESC, methanol extract of Smilax china L.; Bcl-2, B-cell lymphoma 2; t-Bid, truncated BH3 interacting-domain death agonist; Bim, B-cell lymphoma 2 (Bcl-2) interacting mediator of cell death; Bak, Bcl-2 homologous antagonist killer; Bax, Bcl-2-associated X protein; Bcl-x1, B-cell lymphoma-extra large; Mcl-1, induced myeloid leukemia cell differentiation protein; SD, standard deviation.

number of Bcl-2 protein family members associate with the mitochondrial outer membrane, affecting MMP and causing apoptotic cell death (16). Therefore, western blot analysis was used to determine the protein expression levels of Bcl-2 family members. The results indicated that MESC increased t-Bid and Bim protein levels in MC-3 cells, whereas levels of other Bcl-2 family members were unaltered (Fig. 2A and B). A JC-1 assay was then performed to measure MMP and the 
A
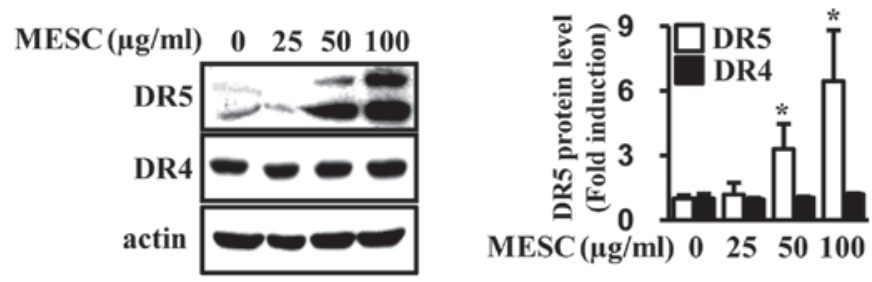

B
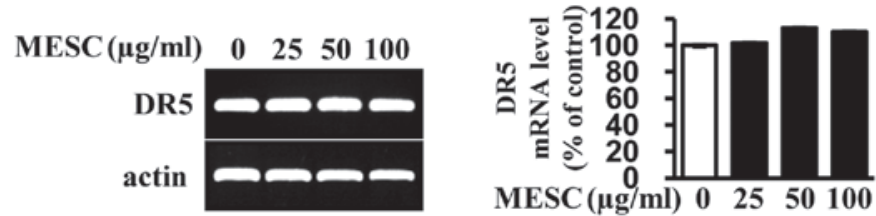

C
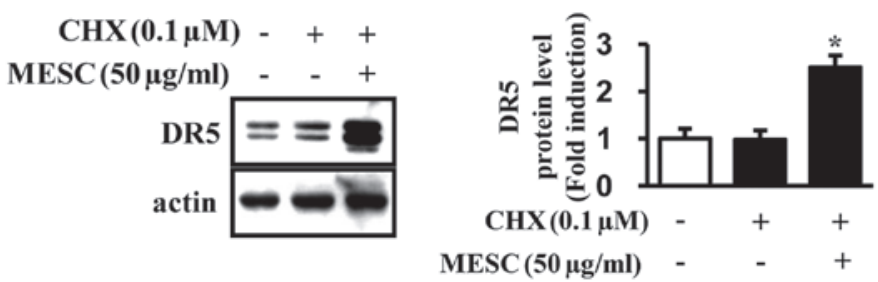

Figure 3. MESC induces DR5 protein through the inhibition of protein degradation. MC-3 cells were treated with dimethylsulfoxide (vehicle control) or various doses of MESC (25, 50 and $100 \mu \mathrm{g} / \mathrm{ml})$ for $24 \mathrm{~h}$. (A) Protein levels of DR5 and 4 were analyzed by western blot analysis, confirmed in triplicate experiments and presented graphically as the mean $\pm \mathrm{SD} ;{ }^{*} \mathrm{P}<0.05$, vs. the vehicle control group. (B) DR5 mRNA levels were confirmed by reverse transcription polymerase chain reaction in MC-3 cells and normalized to actin; data shown in the graphs are presented as the mean \pm SD of triplicate experiments. (C) Following treatment of the cells with $0.1 \mu \mathrm{M}$ CHX in the absence or presence of $50 \mu \mathrm{g} / \mathrm{ml} \mathrm{MESC}$ for $24 \mathrm{~h}$, DR5 expression was analyzed by western blot analysis; the data are presented as the mean \pm SD of triplicate experiments; ${ }^{*} \mathrm{P}<0.05$, vs. the CHX treatment group. MESC, methanol extract of Smilax china L.; $\mathrm{DR}$, death receptor; $\mathrm{SD}$, standard deviation; $\mathrm{CHX}$, cycloheximide.

results indicated that MESC significantly damaged MMP in a dose-dependent manner (Fig. 2C). The involvement of caspase-8 in MESC-induced apoptosis was also determined and data revealed that MESC induced cleavage of caspase- 8 in MC-3 cells (Fig. 2D). Thus, the results suggest that MESC may induce apoptosis through the activation of caspase- 8 and increased levels of t-Bid and Bim, thereby causing alterations in MMP.

MESC increases DR5 through the enhancement of protein stability. To further investigate whether MESC-induced apoptosis is involved in extrinsic pathways associated with DRs, the protein levels of DR4 and 5 were detected. As shown in Fig. 3A, MESC increased the expression of DR5 in a dose-dependent manner compared with the vehicle control but did not affect DR4 expression. The expression levels of DR5 mRNA were also analyzed (by RT-PCR) and the results revealed that DR5 mRNA levels were unchanged (Fig. 3B). To further examine how MESC increases DR5 protein levels, the effects of MESC on protein turnover were investigated using CHX, a protein synthesis inhibitor. The results demonstrated that co-treatment of MESC and CHX significantly increased DR5 protein levels, suggesting that MESC significantly improved DR5 protein stability in MC-3 cells (Fig. 3C).

MESC inactivates the ERK1/2 pathway to induce apoptosis in MC-3 cells. To further investigate whether MESC-induced apoptosis is associated with the MAPK pathway through DRs, the phosphorylation of ERK, JNK, and p38 was analyzed. The results indicated that MESC interrupted the activation of ERK in MC-3 cells but did not induce any changes in the phosphorylation or total expression of JNK and p38 (Fig. 4A and B). To further confirm whether inactivation of ERK was required for the induction of DR5 and apoptosis in MC-3 cells, the ERK inhibitor (PD98059) was used. The results demonstrated that co-treatment with MESC and the ERK inhibitor significantly increased the expression of DR5 in MC-3 cells and that the ERK inhibitor potentiated MESC-induced apoptosis in MC-3 cells (Fig. 4C and D). These results suggest that ERK may be associated with MESC-induced DR5 to induce apoptosis in MC-3 cells.

\section{Discussion}

Naturally-occurring products derived from plants are an abundant source of bioactive constituents that have been important in pharmacology to date. Smilax china L., a common Chinese medicine, has been extensively used for the clinical treatment of syphilis, acute bacillary dysentery, chronic nephritis and cancer. A previous study reported that Smilax china L. exhibited a significant antitumor effect through cell cycle arrest and apoptotic induction in HeLa human cervix carcinoma cells (11). In addition, G1/S cycle arrest and induction of apoptosis have been shown to act as molecular mechanisms of antiproliferative activitiy associated with Smilax china L. (12). However, the anticancer activity of Smilax china L. in MEC 
A

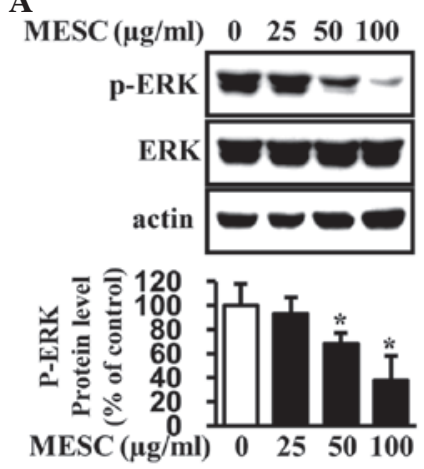

B

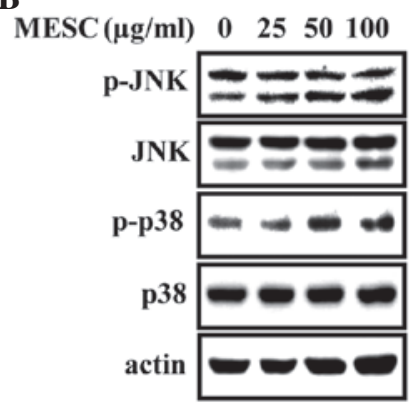

C

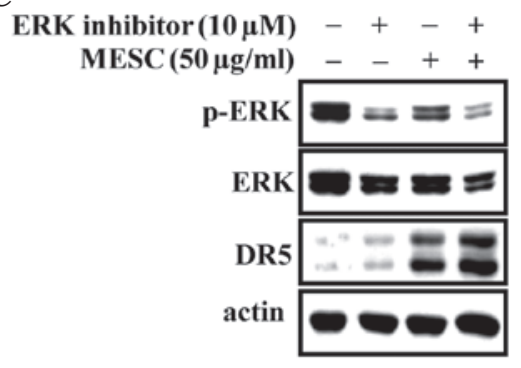

D
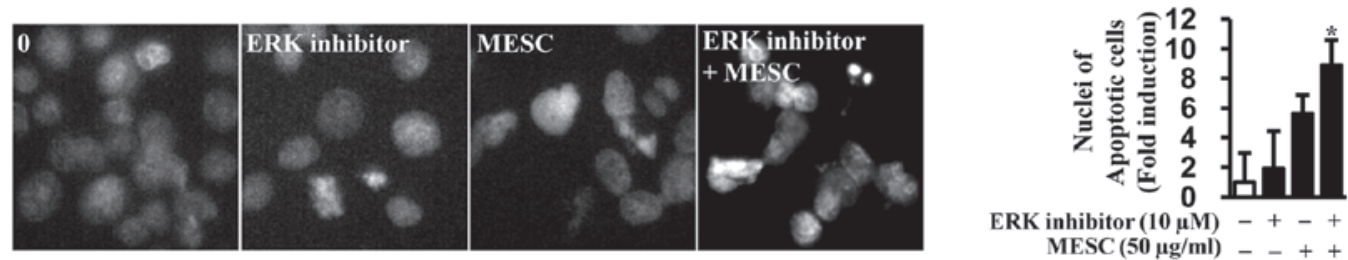

Figure 4. The association between ERK1/2 signaling and MESC-induced apoptosis in MC-3 cells. MC-3 cells were treated with dimethylsulfoxide (vehicle control) or various doses of MESC (25, 50 and $100 \mu \mathrm{g} / \mathrm{ml})$ for $24 \mathrm{~h}$. (A and B) Proteins were extracted to analyze p-ERK, ERK, p-JNK, JNK, p-p38 and p38 expression by western blot analysis. Protein levels were normalized to actin. The expression of p-ERK proteins was confirmed in triplicate with columnar graphs representing the mean $\pm \mathrm{SD}$. "P<0.05, vs. the vehicle control group. (C) Following treatment of the cells with $10 \mu \mathrm{M}$ ERK inhibitor in the absence or presence of $50 \mu \mathrm{g} / \mathrm{ml}$ MESC for $24 \mathrm{~h}, \mathrm{p}$-ERK, ERK and DR5 expression was analyzed by western blot analysis. (D) Fluorescence microscopy images of the DAPI-stanined MC- 3 cells. The apoptotic cells were counted and the data are presented as the mean \pm SD of triplicate experiments; ${ }^{*} \mathrm{P}<0.05$, vs. the MESC treatment group. ERK, extracellular signal-regulated kinase; MESC, methanol extract of Smilax china L.; JNK, c-Jun N-terminal kinase; SD, standard deviation; DR, death receptor.

remains poorly understood. Thus, the present study aimed to explain the ability of Smilax china L. to induce apoptosis and a number of molecular mechanisms. In the present study, MESC was found to decrease cell viability and induce apoptosis in the MC-3 human MEC cell line. Apoptosis is an important mechanism by which the majority of current anticancer agents induce cancer cell death (17). The specific apoptotic pathway activated by an anticancer drug is important not only for predicting its efficacy but also for gaining insight for improved design of therapeutic trials. Therefore, results of the present study suggest that MESC may represent a drug candidate by inducing apoptosis in MC-3 cells.

Apoptosis is tightly regulated by anti-apoptotic and pro-apoptotic molecules, including proteins of the Bcl-2 family $(18,19)$. Therefore, the present study investigated the contribution of $\mathrm{Bcl}-2$ protein family members to MESC-induced apoptosis. Results revealed that MESC resulted in an increase in t-Bid and Bim protein expression, whereas the expression of other family members was not altered. t-Bid and Bim are associated with the mitochondrial outer membrane and are able to disrupt the MMP, resulting in apoptosis in cancer cells. A JC-1 assay was performed to detect the MMP in the present study. The results indicated that MESC significantly damaged MMP, suggesting that the ability of MESC to impair MMP may be associated with MESC-induced apoptosis.

Initiation of apoptosis can be broadly separated into two major pathways, the DR pathway and the mitochondria pathway (20). The DR pathway is mediated by interaction of DRs with their cognate ligands, leading to recruitment of the Fas-associated death domain protein and activation of caspase-8. A number of studies have demonstrated the upregulation of DRs by naturally-occurring chemicals to initiate apoptosis (21-23). In the present study, MESC was found to activate caspase- 8 , indicating that MESC-induced apoptosis may be associated with the extrinsic pathway. Thus, the expression patterns of DR4 and 5 were analyzed and the results demonstrated that MESC upregulated DR5 protein levels in a dose-dependent manner, while DR5 mRNA levels were unchanged. To gain insight into the potential mechanism of MESC regulation of DR5 protein expression, the protein synthesis inhibitor, CHX, was used. Results revealed that co-treatment of CHX and MESC significantly enhanced MESC induction of DR5 protein expression compared with CHX treatment only, suggesting that MESC significantly improves DR5 protein stability in MC-3 cells.

MAPK is important in regulating cell proliferation and cell survival in response to growth stimulation and stress (24). Modulation of the MEK/ERK pathway is associated with specific cellular responses, including regulation of the cell cycle, cell proliferation and apoptosis $(25,26)$. Recently, our group also reported that phenethyl isothiocyanate, from cruciferous vegetables, regulates ERK1/2 or p38, modifying DRs for apoptosis in a number of cancer cell types $(27,28)$. These observations suggest that natural products may affect the MAPK pathway to activate the extrinsic apoptotic pathway. In the present study, MESC was similarly shown to significantly inactivate the ERK pathway whilst the ERK inhibitor (PD980589) potentiated MESC-induced apoptosis through the induction of DR5. These data are consistent with 
previous reports that ERK may be the upstream target of DR5 protein $(29,30)$.

In conclusion, the present study demonstrates that MESC decreases cell viability and induces apoptosis in MC-3 human oral MEC cells. These observations are accompanied by the increase of DR5 through enhanced protein stability, to induce t-Bid and Bim. In addition, MESC inactivates ERK signaling to induce apoptotic cell death. Therefore, this study markedly suggests that Smilax china L. may be a potential chemotherapeutic agent against MEC cells and that ERK inhibition may act as an effective molecule for Smilax china L.-induced apoptosis.

\section{Acknowledgements}

This study was supported by a grant from the Basic Science Research Program through the National Research Foundation of Korea funded by the Ministry of Education, Science and Technology (grant no. 2012003731) and research funds of Chonbuk National University (Jeonju, South Korea) in 2013.

\section{References}

1. Ferlay J, Shin HR, Bray F, Forman D, Mathers C and Parkin DM: Estimates of worldwide burden of cancer in 2008 GLOBOCAN 2008. Int J Cancer 127: 2893-2917, 2010.

2. Johnson NW, Warnakulasuriya S, Gupta PC, et al: Global oral health inequalities in incidence and outcomes for oral cancer: causes and solutions. Adv Dent Res 23: 237-246, 2011.

3. Frankenthaler RA, el-Naggar AK, Ordonez NG, Miller TS and Batsakis JG: High correlation with survival of proliferating cell nuclear antigen expression in mucoepidermoid carcinoma of the parotid gland. Otolaryngol Head Neck Surg 111: 460-466, 1994.

4. Hicks MJ, el-Naggar AK, Byers RM, Flaitz CM, Luna MA and Batsakis JG: Prognostic factors in mucoepidermoid carcinomas of major salivary glands: a clinicopathologic and flow cytometric study. Eur J Cancer B Oral Oncol 30B: 329-334, 1994.

5. Press MF, Pike MC, Hung G, et al: Amplification and overexpression of HER-2/neu in carcinomas of the salivary gland: correlation with poor prognosis. Cancer Res 54: 5675-5682, 1994

6. Handra-Luca A, Ruhin B, Lesty C and Fouret P: P27, SKP2, and extra-cellular signal-related kinase signalling in human salivary gland mucoepidermoid carcinoma. Oral Oncol 42: 1005-1010, 2006

7. Abelson PH: Medicine from plants. Science 247: 513, 1990.

8. Efferth T, Kahl S, Paulus K, et al: Phytochemistry and pharmacogenomics of natural products derived from traditional Chinese medicine and Chinese materia medica with activity against tumor cells. Mol Cancer Ther 7: 152-161, 2008.

9. Khan I, Nisar M, Ebad F, et al: Anti-inflammatory activities of Sieboldogenin from Smilax china Linn.: experimental and computational studies. J Ethnopharmacol 121: 175-177, 2009.

10. Shu XS, Gao ZH and Yang XL: Anti-inflammatory and anti-nociceptive activities of Smilax china $\mathrm{L}$. aqueous extract. J Ethnopharmacol 103: 327-332, 2006.

11. Xu W, Liu J, Li C, Wu HZ and Liu YW: Kaempferol-7-O-betaD-glucoside $(\mathrm{KG})$ isolated from Smilax china L. rhizome induces G2/M phase arrest and apoptosis on HeLa cells in a p53-independent manner. Cancer Lett 264: 229-240, 2008.
12. Li YL, Gan GP, Zhang HZ, et al: A flavonoid glycoside isolated from Smilax china L. rhizome in vitro anticancer effects on human cancer cell lines. J Ethnopharmacol 113: 115-124, 2007.

13. Wu LS, Wang XJ, Wang H, Yang HW, Jia AQ and Ding Q: Cytotoxic polyphenols against breast tumor cell in Smilax china L. J Ethnopharmacol 130: 460-464, 2010.

14. Samanta AK, Huang HJ, Bast RC Jr and Liao WS: Overexpression of MEKK3 confers resistance to apoptosis through activation of NFkappaB. J Biol Chem 279: 7576-7583, 2004.

15. Balmanno K and Cook SJ: Tumour cell survival signalling by the ERK1/2 pathway. Cell Death Differ 16: 368-377, 2009.

16. Li H, Zhu H, Xu CJ and Yuan J: Cleavage of BID by caspase 8 mediates the mitochondrial damage in the Fas pathway of apoptosis. Cell 94: 491-501, 1998.

17. Kim R, Emi M, Tanabe K, Uchida Y and Arihiro K: The role of apoptotic or nonapoptotic cell death in determining cellular response to anticancer treatment. Eur J Surg Oncol 32: 269-277, 2006.

18. Gross A, McDonnell JM and Korsmeyer SJ: BCL-2 family members and the mitochondria in apoptosis. Genes Dev 13: 1899-1911, 1999.

19. Crompton M: Bax, Bid and the permeabilization of the mitochondrial outer membrane in apoptosis. Curr Opin Cell Biol 12: 414-419, 2000

20. Fischer U and Schulze-Osthoff K: New approaches and therapeutics targeting apoptosis in disease. Pharmacol Rev 57: $187-215,2005$.

21. Kok SH, Yeh CC, Chen ML and Kuo MY: Esculetin enhances TRAIL-induced apoptosis through DR5 upregulation in human oral cancer SAS cells. Oral Oncol 45: 1067-1072, 2009.

22. Kim JY, Kim EH, Kim SU, Kwon TK and Choi KS: Capsaicin sensitizes malignant glioma cells to TRAIL-mediated apoptosis via DR5 upregulation and survivin downregulation. Carcinogenesis 31: 367-375, 2010.

23. Yadav VR, Prasad S,Kannappan R, et al: Cyclodextrin-complexed curcumin exhibits anti-inflammatory and antiproliferative activities superior to those of curcumin through higher cellular uptake. Biochem Pharmacol 80: 1021-1032, 2010.

24. Chang L and Karin M: Mammalian MAP kinase signalling cascades. Nature 410: 37-40, 2001

25. Chang F, Steelman LS, Shelton JG, et al: Regulation of cell cycle progression and apoptosis by the Ras/Raf/MEK/ERK pathway (Review). Int J Oncol 22: 469-480, 2003.

26. Agell N, Bachs O, Rocamora N and Villalonga P: Modulation of the Ras/Raf/MEK/ERK pathway by $\mathrm{Ca}(2+)$, and calmodulin. Cell Signal 14: 649-654, 2002.

27. Huong LD, Shin JA, Choi ES, et al: $\beta$-Phenethyl isothiocyanate induces death receptor 5 to induce apoptosis in human oral cancer cells via p38. Oral Dis 18: 513-519, 2012.

28. Huong le D, Shim JH, Choi KH, et al: Effect of $\beta$-phenylethyl isothiocyanate from cruciferous vegetables on grow th inhibition and apoptosis of cervical cancer cells through the induction of death receptors 4 and 5. J Agric Food Chem 59: 8124-8131, 2011.

29. Sung B, Ravindran J, Prasad S, Pandey MK and Aggarwal BB: Gossypol induces death receptor-5 through activation of the ROS-ERK-CHOP pathway and sensitizes colon cancer cells to TRAIL. J Biol Chem 285: 35418-35427, 2010.

30. Belyanskaya LL, Ziogas A, Hopkins-Donaldson S, et al: TRAIL-induced survival and proliferation of SCLC cells is mediated by ERK and dependent on TRAIL-R2/DR5 expression in the absence of caspase-8. Lung Cancer 60: $355-365,2008$ 